\title{
Jejunal linear foreign body obstruction in a three year old female Boerboel
}

\author{
OA Makinde $^{1 *}$, OO Adebayo $^{2}$, AA Adeniyi $^{1} \&$ RA Ajadi $^{1}$ \\ 1. Department of Veterinary Medicine and Surgery, Federal University of Agriculture, Abeokuta, Nigeria \\ 2. Veterinary Teaching Hospital, Federal University of Agriculture, Abeokuta, Nigeria
}

*Correspondence: Tel.: +234 8038058217; E-mail: ayobami2310@gmail.com

\begin{abstract}
Copyright: (C) 2018
Makinde et al. This is

an open-access article published under the terms of the Creative Commons Attribution License which permits unrestricted use, distribution, and reproduction in any medium, provided the original author and source are credited.
\end{abstract}

Publication History: Received: 21-06-2018 Accepted: $30-08-2018$

\begin{abstract}
A three year old Boerboel bitch was presented to the Veterinary Teaching Hospital, FUNAAB with complaint of lethargy and inappetence. Clinical signs observed included abdominal tenderness, watery bloody stool and palpable intestinal mass. Blood sample was obtained for complete blood counts, while transcutaneous abdominal ultrasound and lateral abdominal radiograph were also performed. Result of complete blood count showed severe neutrophilic leukocytosis. Transcutaneous abdominal ultrasound revealed dilated bowel loop containing a hyperechoic structure in the lumen, while radiographic contrast examination of the intestine revealed distended bowel loop and a radiopaque foreign body in the jejunum. Based on the findings, a tentative diagnosis of intestinal obstruction was made and an exploratory laparotomy was scheduled. After pre-anaesthetic medications with $3 \mathrm{mg} / \mathrm{kg}$ intramuscular injection of $5 \%$ tramadol, $0.04 \mathrm{mg} / \mathrm{kg}$ intramuscular injection of $0.1 \%$ atropine and $0.5 \mathrm{mg} / \mathrm{kg}$ intramuscular injection of $2 \%$ Xylazine, anaesthesia was induced with a loading dose of $4 \mathrm{mg} / \mathrm{kg}$ intravenous injection of $1 \%$ propofol and maintained with constant infusion of propofol at the rate of $0.12 \mathrm{mg} / \mathrm{kg} / \mathrm{min}$. During laparotomy, the obstructed region was identified and enterotomy revealed the foreign body to be an ingested plastic bottle cap. Following removal of the foreign body, moderate inflammation of the segment of the intestine around the obstruction site was observed, necessitating the need for resection of the intestine to be performed and anastomosis using an end to end technique. The dog recovered without any complications after one week of postoperative antibiotic therapy and fluid administration. Prompt diagnosis of intestinal foreign body in dogs followed by appropriate selection of anaesthetic protocol and surgical technique with adequate intensive post-operative follow-up is essential for successful management.
\end{abstract}

Keywords: Dog, Foreign-body, Jejuno-ileal, Intestine, Obstruction

\section{Introduction}

Intestinal foreign bodies are commonly encountered in companion animal practice and may present with a variety of clinical signs depending on the location, the degree and the duration of the obstruction resulting from them (Aronson et al., 2000; Papazoglou et al., 2003). Foreign bodies commonly reported include bones, migrating wires (Padalkar et al., 2017), sterile injection port (Allan, 2015) and female tampon (Yang et al., 2018). Intestinal obstruction may result in disturbances of fluid balance, acid-base status and serum electrolyte concentrations (Boag et al., 2005). This may be due 
to induction of haemorrhagic diarrhoea as a result of bacteria localization in the obstructed end of the GIT or release of histamines by the intestinal mucosa cells (Yang et al., 2018) Intestinal foreign bodies could be linear or nonlinear. Linear foreign bodies are reported to be associated with higher mortality than non-linear foreign bodies in cats and often results in plication of the small intestine (Allan, 2015). Non-linear intestinal foreign bodies can cause partial or complete obstruction leading to compromise of blood supply to the intestinal segment by luminal distention leading to intestinal wall oedema and progressive necrosis (Hayes, 2009).

Linear intestinal foreign bodies have been reported to be higher in cats (50-61\%) (Bebchuk, 2002) than dogs (36\%) (Boag et al., 2005). Common anchorage point for linear foreign bodies in dogs is the pylorus, distal duodenum and the proximal jejunum (Hayes, 2009). Other sites are mid-jejunum and jejuno-ileum (Allan, 2015) Reported linear foreign bodies in dogs include fabrics, plastic, thread, sewing needles, wires and textile materials (Hayes, 2009; Pratt et al., 2014; Allan, 2015; Padalkar et al., 2017)

The major challenges with the management of intestinal foreign bodies are early diagnosis of the condition, risk associated with anaesthesia of patient with compromised electrolyte and acid base status. Surgical management and wound healing are compromised by intestinal wall viability, intraluminal bacterial overgrowth, ileus and hypoproteinaemia (Ralphs et al., 2003). Enteric wound breakdown and leakage are the most serious and catastrophic complications of surgery on the gastrointestinal tract (Hayes, 2009). Survival rate for intestinal linear foreign bodies was reported to be about $80 \%$ (Hayes, 2009). This report presents the diagnosis and surgical management of jejunal linear foreign body in a three-year-old Boerboel bitch.

\section{Case Management}

Signalment and history

A three-year-old Boerboel bitch was presented to the VTH, FUNAAB with complaint of lethargy and inappetence which had persisted for three days prior to presentation. Physical examination revealed moderate fever $\left(40.6^{\circ} \mathrm{C}\right)$ and congested ocular mucous membrane. Superficial lymph nodes of the dog were not enlarged. However, there was moderate ventral distension of the mid abdomen. Palpation of the abdomen revealed moderate distension of the bowel loops from which a straw coloured gritty material was aspirated.

Blood was obtained from the cephalic vein for complete blood counts. In addition, microscopic evaluation of the bowel aspirate was carried out. Also, transcutaneous abdominal ultrasound and contrast radiographic examination of the intestine was performed.

\section{Results}

Microscopic examination of the bowel aspirate showed that it had the composition of faecal material. Result of complete blood count is presented in Table 1 . Transcutaneous abdominal ultrasound revealed dilated bowel loop (Plate I) containing a hyperechoic structure in the lumen (Plate II). In addition, contrast examination of the intestine also revealed distended bowel loop and a radiopaque foreign body in the jejunum (Plate III). Based on the findings, a tentative diagnosis of intestinal obstruction was made and an exploratory laparotomy was scheduled.

\section{Case management}

Food and water was withheld from the dog for 24 hours prior to surgery. The hair on the ventral abdomen was shaved from around the xyphoid

Table 1: Complete blood count of a three-year-old Boerboel bitch with jejuno-ileal foreign body

\begin{tabular}{lll}
\hline Hematological parameters & Patient's value & Normal range \\
\hline PCV (\%) & 30 & $29-55$ \\
WBC (× 1000) & 13.2 & $5.9-16.6$ \\
Neutrophil (\%) & 72 & $51-84$ \\
Eosinophil (\%) & 0 & $0-9$ \\
Basophil (\%) & 0 & $0-1$ \\
Lymphocyte (\%) & 26 & $8-38$ \\
Monocyte (\%) & 02 & $1-9$ \\
\hline
\end{tabular}




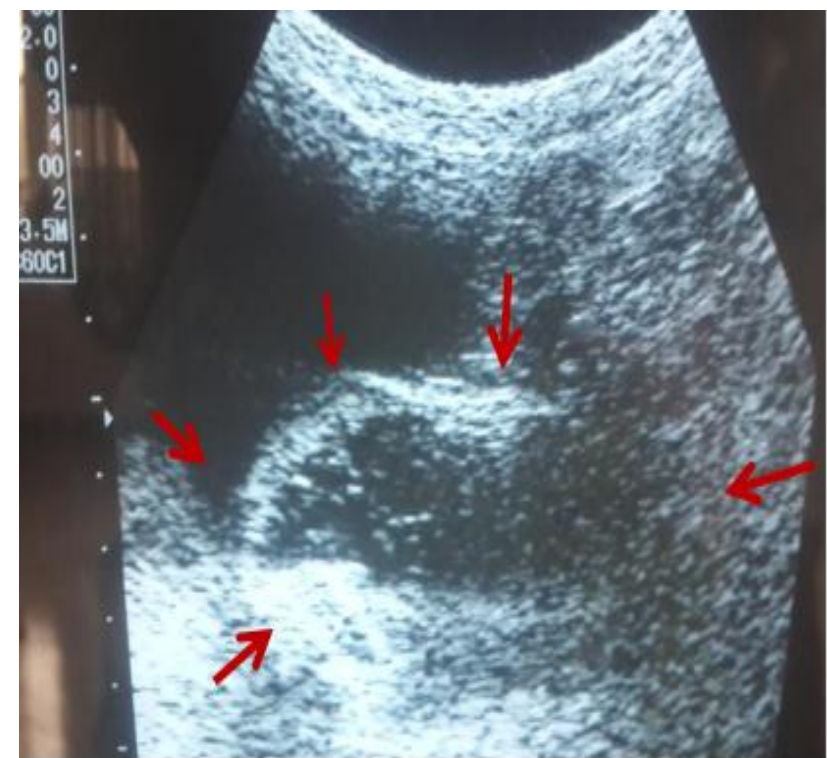

Plate I: Transcutaneous abdominal ultrasound of a three-year-old Boerboel bitch showing dilated bowel loop (arrows)

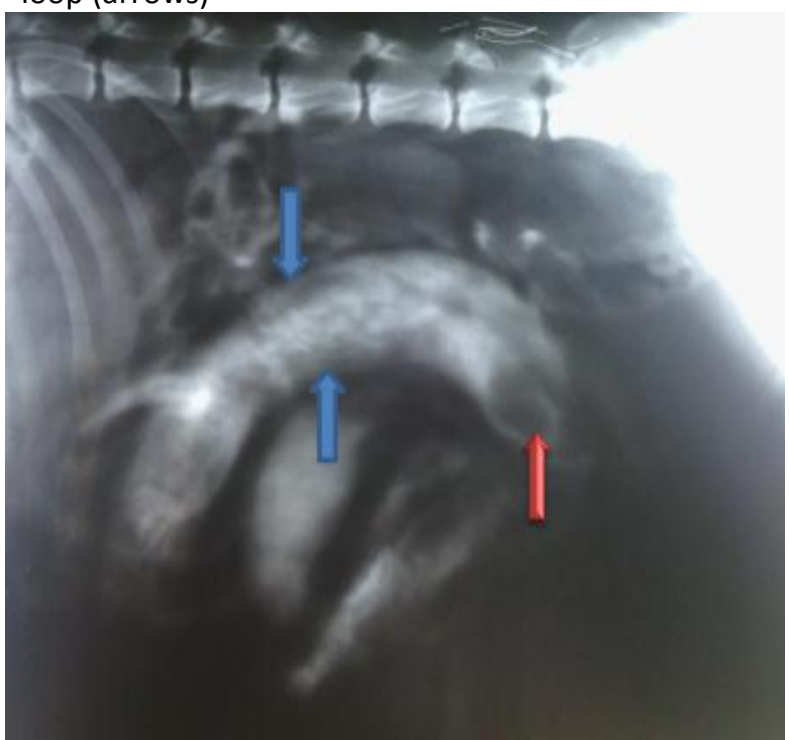

Plate III: Contrast examination of the intestine showing distended bowel loop (blue arrows) with an obstruction in the jejunum (red arrow)

cartilage to the prepubic tendon. Thereafter, the skin was scrubbed and disinfected with $0.05 \%$ Chlorhexidine solution. Following anaesthesia, the dog was positioned in dorsal recumbency and the legs secured to the edges of the table using gauze bandage. Finally, the abdomen was disinfected again and sterile drapes were applied. The dog was premedicated with $3 \mathrm{mg} / \mathrm{kg}$ intramuscular injection of $5 \%$ tramadol (Tramadol ${ }^{\circledR}$, Gland Pharma, India) and thirty minutes later with a combination of

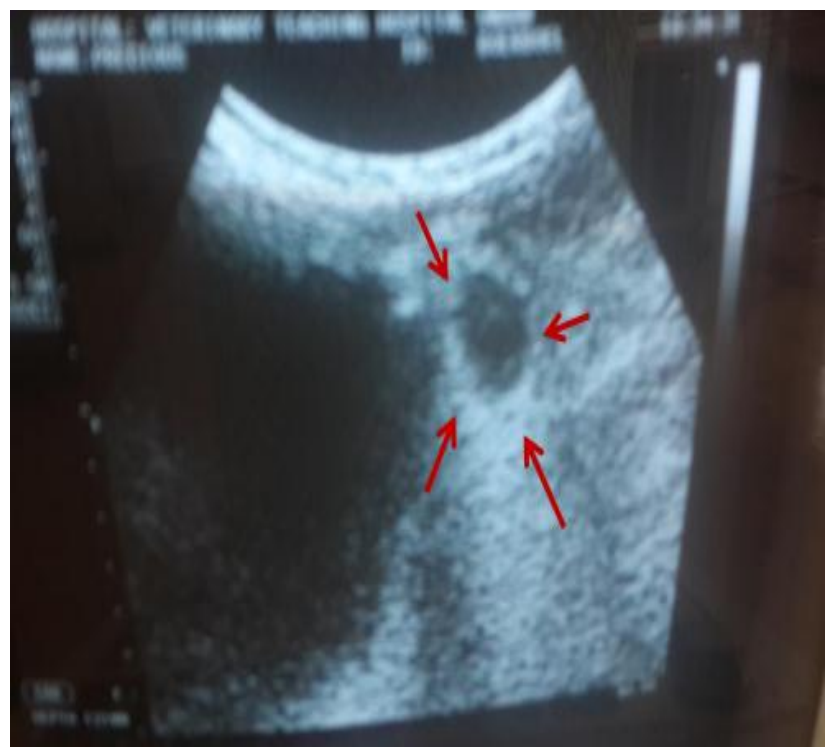

Plate II: Transcutaneous abdominal ultrasound of a three-year-old Boerboel bitch with intestinal foreign body (arrow)

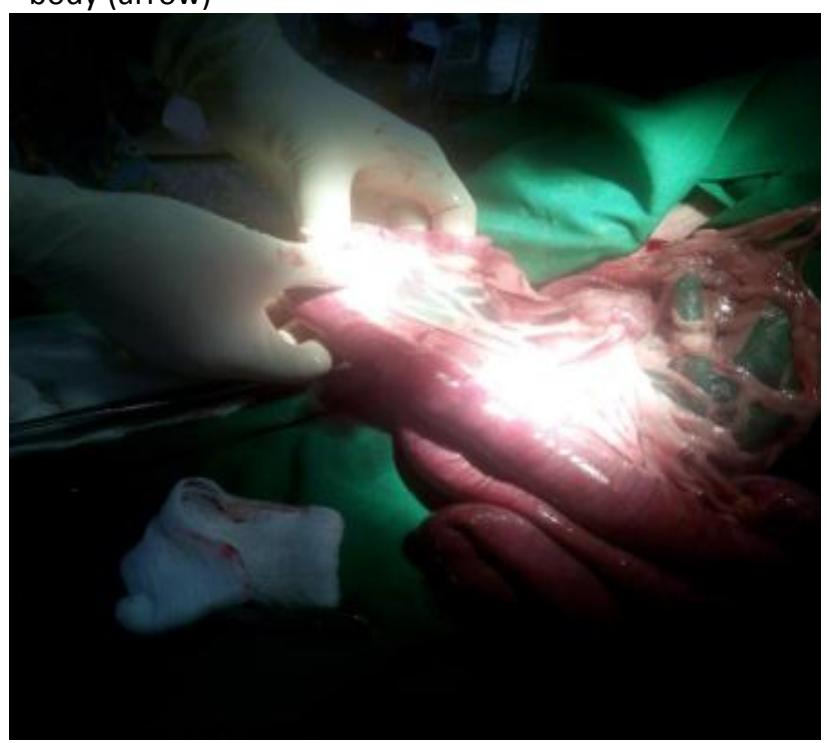

Plate IV: Laparotomy in a three-year-old Boerboel bitch showing the exteriorized intestinal segment

$0.04 \mathrm{mg} / \mathrm{kg}$ intramuscular injection of $0.1 \%$ atropine sulphate (Atocan $^{\circledR}$, Sishui Xierkang Pharma, China) and $0.5 \mathrm{mg} / \mathrm{kg}$ intramuscular injection of $2 \%$ Xylazine Hydrochloride (Xylazine $20 \mathrm{Inj}$, Kepro, Holland). Venous access was secured and circulating blood volume was maintained with Lactated Ringers solution at the rate of $5 \mathrm{~mL} / \mathrm{min}$. Anaesthesia was induced with a loading dose of $4 \mathrm{mg} / \mathrm{kg}$ intravenous injection of $1 \%$ propofol (Diprivan, ICl - Zeneca Pharmaceuticals) and maintained with constant 


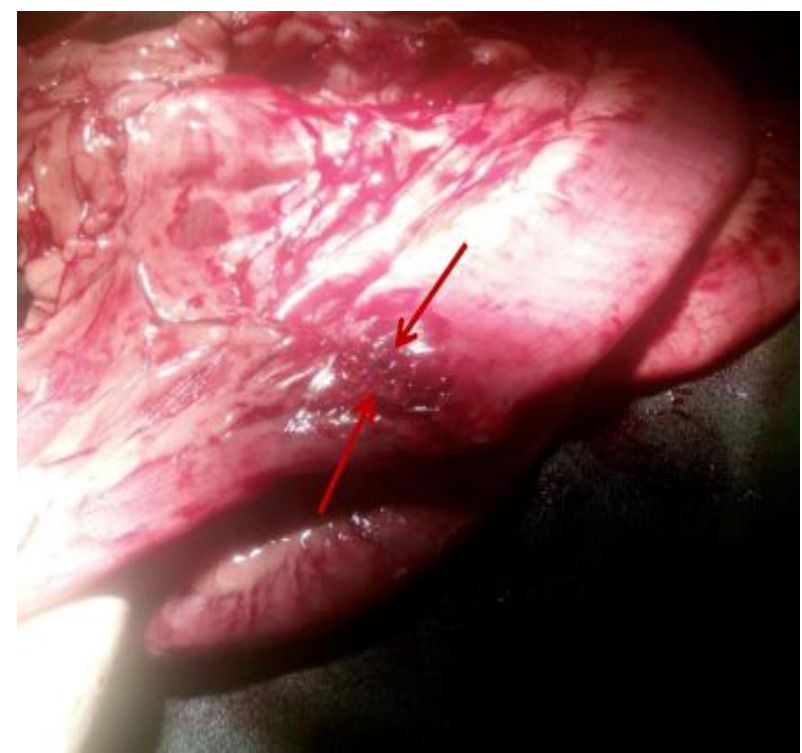

Plate V: Anastomosed segment of the intestine (arrows) of a three-year-old Boerboel bitch following resection to remove a foreign body

infusion of propofol at the rate of $0.12 \mathrm{mg} / \mathrm{kg} / \mathrm{min}$. Following anaesthesia, the dog was positioned in dorsal recumbency and the legs were secured to the edges of the table using gauze bandage. Finally the abdomen was disinfected again and sterile drapes were applied. A ventral midline incision was made on the skin. After careful dissection of the subcutaneous tissue, the linea alba was located and a stab incision was made on it. The incision was then lengthened using a pair of myo-scissors to gain access into the abdominal cavity. The intestine was then exteriorized and explored (Plate IV). After locating the foreign body, it was observed that there was moderate inflammation of the segment of the intestine around it thus the intestinal segment was resected and anastomosed using an end-to-end anastomosis technique (Plate V). The foreign body (Plate VI) was observed to be a plastic bottle cap. The anastomosed intestine was rinsed and then returned into the abdominal cavity and $5 \mathrm{~mL}$ of Penicillin-streptomycin (PenStrep , Kepro, Holland) was infilterated. The laparotomy incision was then closed in three layers. The linea alba was closed with double rows of simple continuous suture pattern using size 1 catgut. The subcutaneous tissue was closed with subcorticular pattern using size 0 chromic catgut, while the skin was closed with horizontal mattress suture pattern with size 0 polyester.

During post-operative management, tramadol injection at $4 \mathrm{mg} / \mathrm{kg}$ was administered intramuscularly. In addition, $4 \mathrm{~mL}$ of

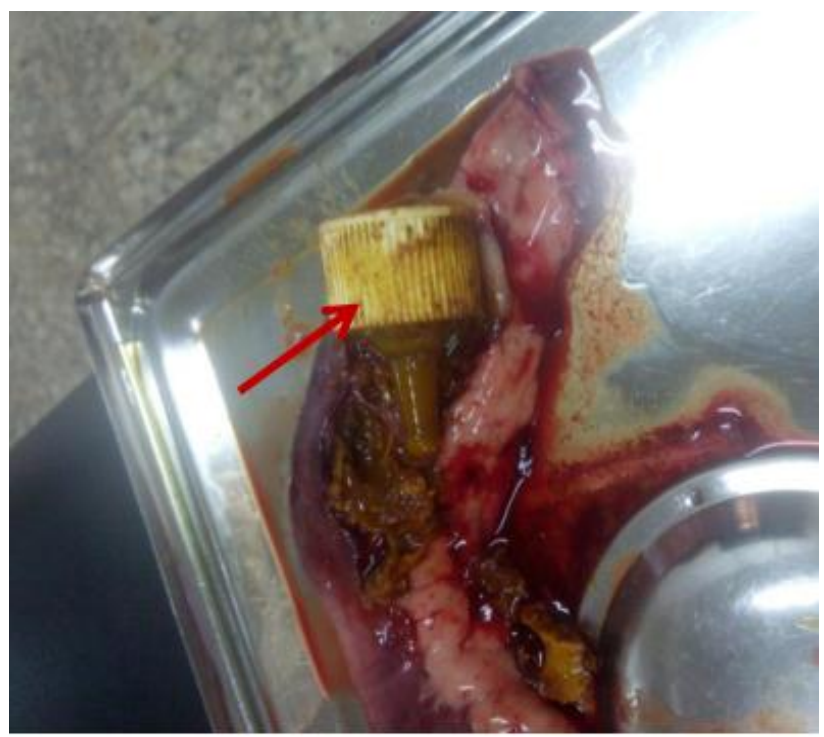

Plate VI: Plastic bottle cap removed from the intestine of a three-year-old Boerboel bitch

penicillin/streptomycin injection was administered intramuscularly for one week. The dog was monitored till recovery and additional $1000 \mathrm{mLs}$ of Lactated Ringers solution was administered intravenously. The dog recovered without any complication

\section{Discussion}

Gastrointestinal foreign bodies are commonly encountered in small animal practice. Due to indiscriminate eating habits, foreign bodies are more common in dogs than cats (Hayes, 2009). Most ingested foreign bodies pass uneventfully through the gastrointestinal tract, however, some may result in signs such as regurgitation, vomiting, diarrhea, or signs of small intestinal obstruction thus alerting the owner to note that something is wrong. The only sign presented by this dog is inappetence. It is interesting to note that in spite of the small intestine distension, the dog did not present with regurgitation. This might be as a result of the incomplete obstruction of the intestinal segment which still allows ingesta to pass through the bowel. The severe distension of the intestine despite the fact that the dog was not eating may be due to absorption of fluid into the intestinal lumen occasioned by endotoxin secretion (Boag et al., 2005).

A technique of single enterotomy removal of linear foreign bodies has been reported and used with good success (Hayes, 2009), many chronic foreign objects may not be safely manipulated due to severe 
compromise of the local gastrointestinal segment. This explains the choice of resection and anastomosis technique over enterotomy. The surgical technique used also allows for the removal of devitalized intestinal segments. However, the technique is more time consuming and with higher risk of leakage.

Several techniques have been reported for the anastomosis of the intestinal segments following resection. These include end to end, end to side, side to side and floating technique (Ralphs et al., 2003). The technique of choice depends on the length of the intestine that is resected and the diameter of the two ends. An end to end anastomosis was performed using Lembert suture pattern because the resected ends were of the same diameter.

Survival rate for surgical removal of intestinal foreign body has been reported to be about 80 percent (Hayes, 2009). The major causes of mortality include rupture and peritonitis, post-operative hypovolemic or endotoxic shock and anaesthetic complication (Ralphs et al., 2003; Hayes, 2009).The dog recovered without any complication. In conclusion, prompt diagnosis of intestinal foreign body in dogs followed by appropriate selection of anaesthetic surgical technique with adequate intensive post-operative follow-up is essential for successful management.

\section{References}

Allan RM (2015). Injection port aids linear foreign body removal in a cat. Veterinary Record Case Report; doi.org/10.1136/vetreccr2015-000217.

Aronson LR, Brockman DJ \& Brown DC (2000). Gastrointestinal emergencies. Veterinary Clinics of North America: Small Animal Practice. 30(3): 555-579.
Bebchuk TN (2002). Feline gastrointestinal foreign bodies. Veterinary Clinics of North America: Small Animal Practice. 32(4): 861-880.

Boag AK, Coe RJ, Martinez TA \& Hughes D (2005). Acid-base and electrolyte abnormalities in dogs with gastrointestinal foreign bodies. Journal of Veterinary Internal Medicine. 19(6): 816-821.

Hayes G (2009). Gastrointestinal foreign bodies in dogs and cats: a retrospective study of 208 cases. Journal of Small Animal Practice 50(11): 576- 583.

Padalkar M, Savage M \& Cohen EB (2017). Small Intestinal Intramural Hematoma secondary to a migrating wire foreign body in a dog. Veterinary Radiology and Ultrasound, doi: 10: 1111/vru.12485.

Papazoglou LG, Patsikas MN \& Rallis T (2003). Intestinal foreign bodies in dogs and cats. Compendium on Continuing Education for the Practicing Veterinarian. 25(11): 830843.

Pratt CL, Reincke EC \& Drobatz KJ (2014). Sewing needle foreign body ingestion in dogs and cats: 65 cases (2000 - 2012). Journal of American Veterinary Medical Association, 245(3):302-308.

Ralphs SC, Jessen CR \& Lipowitz AJ (2003). Risk factors for leakage following intestinal anastomosis in dogs and cats: 115 cases (1991-2000). Journal of American Veterinary Medical Association. 223(1): 7377.

Yang S, Kim J, Jeong S \& Han H (2018). Acute hemorrhagic diarrhea syndrome associated with contaminated foreign bodies (used feminine hygiene products) in a Golden Retriever dog. The Journal of Veterinary Medical Science, 80(4): $629-633$. 